\title{
Unstable periodic orbit detection for ODEs with periodic forcing
}

\author{
J.H.B. Deane and L. Marsh \\ Department of Mathematics \& Statistics, University of Surrey \\ Guildford, Surrey, GU2 7XH, UK \\ Email: J.Deane@eim.surrey.ac.uk
}

\begin{abstract}
The Davidchack-Lai iterative scheme for the complete detection of unstable periodic orbits (UPOs) in maps is applied to a second order, nonlinear ODE with periodic forcing. The modifications to the scheme required to apply it to ODEs are detailed before the results for a particular example, the varactor equation, are given.
\end{abstract}

PACS code: $05.45 \mathrm{Pq}$

Keywords: unstable orbit detection, numerics, chaos

\section{Introduction}

UPOs have been recognised as one of the fundamental building blocks of invariant sets in chaotic dynamical systems, since many variables of physical interest can be related to the dynamical properties of the set of UPOs, infinite in number, embedded in the chaotic set $[1,2,3]$. Detection of a complete set of UPOs, especially at high periods, is difficult since the number of UPOs grows exponentially and the basin of attraction of each shrinks as the period increases. Recently, Davidchack and Lai (DL) [4, 5] presented a method for the complete detection of UPOs which converges rapidly and is only limited by computer precision and time. Although the DL method could in principle be applied to ODEs, it has so far $[4,5]$ only been applied to maps, in particular the Hénon map, whose UPOs were previously documented in [1], and the Ikeda-Hammel-Jones-Moloney map, whose UPOs were previously unknown. Now, according to $[4,5]$, the method is also applicable to ODEs, although no examples are given either there or anywhere else in the literature, as far as the authors are aware. Hence, in this paper, we apply the DL method to a particular case of the non-autonomous ODE

$$
\ddot{x}+\gamma \dot{x}+F(x)=f(t)
$$

where $F(x)$ is a polynomial of degree $>1$ and $f(t)$ is a periodic function with period $T$. 


\section{The DL iterative scheme for mappings}

Let $\mathbf{x} \in \mathbb{R}^{N}$ and $\mathbf{x}_{n+1}=\mathbf{f}\left(\mathbf{x}_{n}\right)$ be an $N$ dimensional map. Any periodic orbit of period $p$ can be detected as zeros of the function $\mathbf{g}(\mathbf{x})=\mathbf{f}^{(p)}(\mathbf{x})-\mathbf{x}$, where $\mathbf{f}^{(p)}(\mathbf{x})$ is the $p$-th iteration of the map $\mathbf{f}(\mathbf{x})$. By choosing an initial point and computing successive corrections, zeros of $\mathbf{g}(\mathbf{x})$ can be found. These corrections $(\delta \mathbf{x})$ can, for instance, be calculated by using the DL iterative scheme, which is given by

$$
\mathbf{x}_{\text {new }}=\mathbf{x}_{\text {old }}+\delta \mathbf{x}=\mathbf{x}_{\text {old }}+[\mathbf{I} \beta g(\mathbf{x})-\mathbf{C J}(\mathbf{x})]^{-1} \mathbf{C g}(\mathbf{x}),
$$

where $\mathbf{J}(\mathbf{x})=\frac{\partial \mathbf{g}}{\partial \mathbf{x}}$ is the Jacobian matrix, $\mathbf{I}$ is the unit matrix, $\mathbf{C}$ is an $N \times N$ matrix with elements $C_{i j} \in\{0, \pm 1\}$ such that each each row or column contains exactly one nonzero element; $g(\mathbf{x}) \equiv\|\mathbf{g}(\mathbf{x})\| \geq 0$ is the magnitude of the vector $\mathbf{g}$ and $\beta>0$ is an adjustable parameter. This method combines both the Newton-Raphson (NR) [6] and the Schmelcher-Diakonos (SD) [7] methods. In the NR method, the corrections are calculated from the set of $N$ linear equations $-\mathbf{J}(\mathbf{x}) \delta \mathbf{x}=\mathbf{g}(\mathbf{x})$, whereas in the SD method, the corrections are given by $\delta \mathbf{x}=\lambda \mathbf{C g}(\mathbf{x})$, where $\lambda$ is a small positive number. The advantage of the NR method is that it has rapid convergence, approximately doubling the number of significant digits on every iteration (quadratic convergence), provided that the initial point is within the linear neighbourhood of the solution - roughly speaking, the region in which the function can be well approximated linearly. On the other hand, the advantage of the SD method is that the basin of attraction of each UPO extends far beyond its linear neighbourhood, so most initial points converge to some UPO. With the DL method, the function $g(\mathbf{x})$ in the vicinity of any UPO tends to zero, so that the NR method is restored and quicker convergence is obtained. Further away from the solution and with sufficiently large values of $\beta$, the DL method is similar to the SD method and thus largely preserves its global convergence property.

In implementing the DL method, we follow [4] in selecting initial points for finding UPOs of period $p$ using orbit points of periods $p-1$ and $p+1$, which turns out to be better than using randomly selected points or points on a periodic grid.

\section{From map to ODE}

Applying the DL method to a non-autonomous ODE requires two changes to the DL scheme as applied to maps. First, the map itself needs to be replaced with an ODE solver which integrates the ODE over one period, $T$, of $f(t)$. Second, the Jacobian cannot be expressed in closed form either, so this, too, must be estimated numerically.

The first modification involves replacing the map function by an ODE solver which integrates the ODE from initial condition $\left(x_{n}, y_{n}\right)$ at $t=t_{0}$ to find $x_{n+1}, y_{n+1}$, the solution at $t=t_{0}+T$. One way to do this would use a standard numerical ODE solver, and although this would work in principle, speed is critical since the map $\mathbf{f}$ has to be computed a large number of times. Hence, it is desirable to use an ODE solver that is as fast as possible, and to produce the results reported here we used a method based on Taylor series, similar to that outlined in [8], also known as the cell-to-cell mapping technique. In brief, the solution 
of the ODE is expanded in a power series around a point $t=t_{0}$, and a suitably modified ratio test applied to the high-order coefficients of the series. The test gives an estimate of the radius of convergence of the series, $R$, and so we can compute the solution accurately at, say, $t=t_{0}+R / 2$. This is effectively numerically-implemented analytical continuation. In practice, $R$ can be quite large and so the ODE can be solved in correspondingly large time steps, leading to an increase in speed by a factor of about 10-50. Full details are given in $[9]$.

The second modification entails finding an alternative method to estimate the Jacobian when the map is not available explicitly, and numerical differentiation is therefore required. This can be carried out by using the three point formula [10] $f^{\prime}\left(x_{0}+\mu h\right)=$ $\left[(2 \mu-1) f_{-1}-4 \mu f_{0}+(2 \mu+1) f_{1}\right] /(2 h)$, where $f_{\mu}=f\left(x_{0}+\mu h\right)$, with $\mu$ not necessarily integral, and $h$ is the stepsize. Since the derivative is required at the point $x_{0}, \mu=0$, which results in the simple first difference formula $f^{\prime}\left(x_{0}\right)=\left[f_{1}-f_{-1}\right] /(2 h)$. Perhaps surprisingly, this was found to be adequate in practice.

\section{Worked example}

An example is now considered in the form of the differential equation (1) with $F(x)=x^{2}$, $\gamma=0.01$ and $f(t)=3+2.5 \sin t$, which describes the biased driven RL-diode circuit [11]. This is a good candidate for testing purposes for two reasons. First, solutions to this differential equation have been proved to blow up in finite time for initial conditions in an infinite-area subset of the phase plane [12], and this places useful limits on the regions where UPOs can be expected to be found. Second, the existence of Melnikov chaos has been proven [13] for solutions to this ODE, hinting that a nontrivial set of UPOs might be expected. Our results were obtained using the program detailed in [11], and they are considered to be close to complete, at least for $p<10$. Even with the fast ODE solver described, the computer time taken to compute these results was of the order of several weeks.

\begin{tabular}{|r|r|r|r||r|r|r|r||r|r|r|r|}
\hline$p$ & $N_{p}$ & $N_{s}$ & $\beta_{\min }$ & $p$ & $N_{p}$ & $N_{s}$ & $\beta_{\min }$ & $p$ & $N_{p}$ & $N_{s}$ & $\beta_{\min }$ \\
\hline 1 & 4 & 1 & 10 & 5 & 7 & 1 & 225 & 9 & 34 & 0 & Unknown \\
2 & 2 & 0 & 10 & 6 & 11 & 0 & 1025 & 10 & $47^{*}$ & 0 & Unknown \\
3 & 3 & 1 & 10 & 7 & 16 & 0 & Unknown & 11 & $44^{*}$ & 0 & Unknown \\
4 & 3 & 0 & 30 & 8 & 27 & 0 & Unknown & 12 & $50^{*}$ & 0 & Unknown \\
\hline
\end{tabular}

Table 1: Number of UPOs $N_{p}$ of period $p$ for the differential equation $(1)$ with $F(x)=x^{2}$, $\gamma=0.01$ and $f(t)=3+2.5 \sin t . N_{s}$ is the number of stable periodic orbits found. $\beta_{\min }$ is the minimal $\beta$ value which results in the detection of all $N_{p}$ period- $p$ UPOs. The asterisk next to certain $N_{p}$ values indicates that these sets might be incomplete.

These results are summarised in Table 1 , where $N_{p}$ is the number of UPOs detected, $N_{s}$ is the number of stable periodic orbits found and $\beta_{\min }$ is the minimal $\beta$ value which yields all 
$N_{p}$ UPOs. $N_{p}$ values for periods 10, 11 and 12 were found in an early run of the program, but it is believed that these are incomplete sets; evidence for this can be seen in figure (1), which is a graph of $\ln N_{p}$ against $p$. For $2 \leq p \leq 10$, the $\ln N_{p}(p)$ relationship appears to be well approximated by a straight line. Outside this range of $p$, the graph suggests that there may be some UPOs missing. These undetected UPOs could result from a single missing UPO of period 10 or 11, which in turn would mean that initial conditions are absent for higher periods. Figure (1) suggests that the topological entropy, defined [7] by $S_{T}=\lim _{p \rightarrow \infty} \frac{1}{p} \ln N_{p} \approx 0.42$.

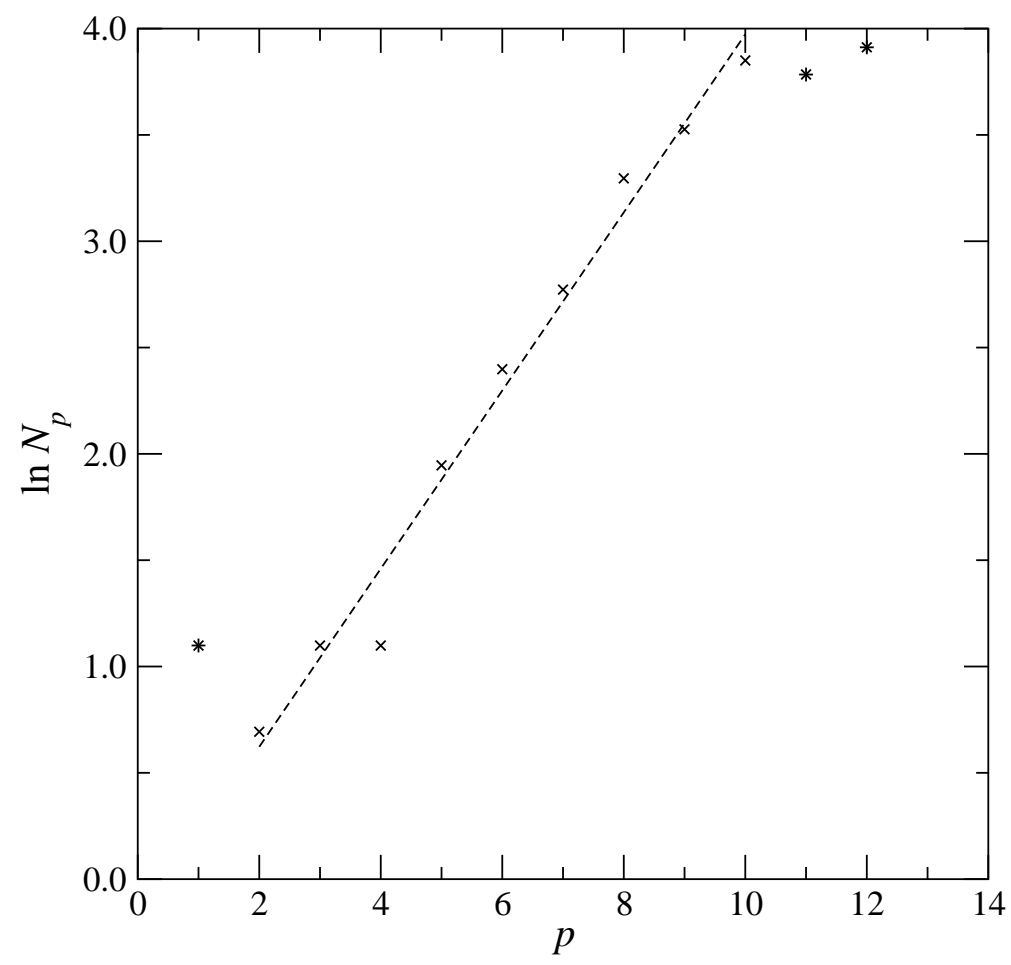

Figure 1: The graph of $\ln N_{p}$ versus $p$ from Table (1) and a least squares fit (dashed line) for $p$ in the range $p=2 \ldots 10$. The points used in the fit are shown as $\times$ and those not used, as $*$. The least squares fit line is $\ln N_{p} \approx-0.22+0.42 p$.

The UPOs given in table (1) were found using the entire set of eight $\mathbf{C}$ matrices with the majority of UPOs being found using just the matrices $\mathbf{C}_{1} \ldots \mathbf{C}_{5}$, these being

$$
\mathbf{C}_{1}=\left[\begin{array}{ll}
1 & 0 \\
0 & 1
\end{array}\right], \mathbf{C}_{2}=\left[\begin{array}{rr}
-1 & 0 \\
0 & 1
\end{array}\right], \mathbf{C}_{3}=\left[\begin{array}{rr}
1 & 0 \\
0 & -1
\end{array}\right], \mathbf{C}_{4}=\left[\begin{array}{rr}
0 & -1 \\
-1 & 0
\end{array}\right], \mathbf{C}_{5}=\left[\begin{array}{ll}
0 & 1 \\
1 & 0
\end{array}\right] .
$$

Initial conditions for orbits of period $p$ were taken from the orbit points of UPOs of period $p-1$ only. The UPOs of periods 1 and 2 were found by searching on a $20 \times 20$ grid of initial conditions uniformly spaced over $x \in[-1.2,1.1], y \in[-1.5,2]$. Three stable solutions with periods 1,3 and 5 were also found. 

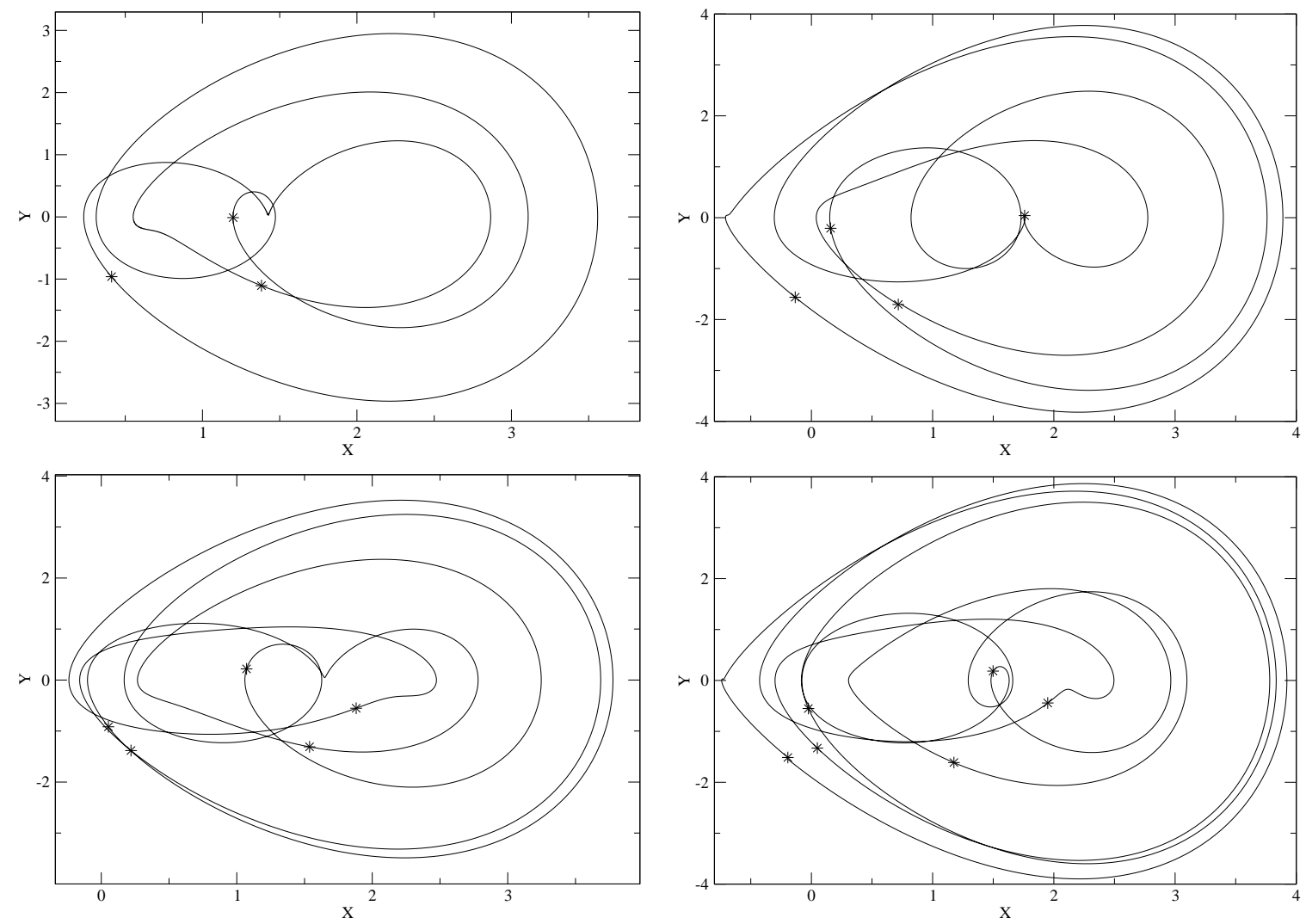

Figure 2: Four coexisting UPOs of the differential equation (1) with $F(x)=x^{2}, \gamma=0.01$ and $f(t)=3+2.5 \sin t$. Solutions shown are period-3 (top left), period-4 (top right), period5 (bottom left), and period-6 (bottom right). The asterisks denote the positive-going zero crossings of $f(t)$, i.e. the points $\mathbf{x}(0), \mathbf{x}(2 \pi), \mathbf{x}(4 \pi), \ldots$

Phase plane diagrams of several unstable solutions are shown in figure (2), with the asterisks denoting the positive-going zero crossings of $f(t)$, i.e. $\mathbf{x}(0), \mathbf{x}(2 \pi), \mathbf{x}(4 \pi), \ldots$.

Some preliminary calculations were also carried out for Duffing's equation in the form $\ddot{x}+0.25 \dot{x}+x^{3}-x=0.35 \sin t$, a widely-studied example of an ODE that, for these parameter values, displays chaotic solutions. This is a much more difficult example because UPOs can now exist in principle anywhere in the phase plane. Hence, it is considerably harder to guarantee that all UPOs, even those of low periods, have been found. Initial results indicate that $N_{1}=3, N_{2}=10, N_{3}=42$ and $N_{4}=84$.

\section{Conclusions}

We have taken an algorithm for the detection of unstable periodic orbits (UPOs) in discrete dynamical systems, and, we believe for the first time, applied it to a continuous dynamical system, in this case a nonlinear differential equation. A particular ODE was chosen as an example, which was then worked out in detail. The algorithm requires that the differential 
equation be solved over long time intervals, and in order to speed up the numerics to a point where the computation feasible, a fast ODE solving algorithm was devised for the worked example. Sets of UPOs have been detected for the problem considered and these are believed to be complete for periods 1 to at least 9 .

\section{References}

[1] D. Auerbach, P. Cvitanović, J-P. Eckmann, G. Gunaratne and I. Procaccia, Exploring chaotic motion through periodic orbits, Physical Review Letters, vol. 58, no. 23, pp. 2387-2389 (1987)

[2] C. Grebogi, E. Ott and J.A. Yorke, Unstable periodic orbits and the dimension of multifractal chaotic attractors, Physical Review A, vol. 37, no. 5, pp. 1711-1724 (1988)

[3] Y-C. Lai, Y. Nagai and C. Grebogi, Characterization of the natural measure by unstable periodic orbits in chaotic attractors, Physical Review Letters, vol. 79, no. 4, pp. 649-652 (1997)

[4] R.L. Davidchack and Y-C. Lai, Efficient algorithm for detecting unstable periodic orbits in chaotic systems, Phys. Rev. E, vol. 60, no. 5, pp. 6172-6175 (1999)

[5] R.L. Davidchack, Y-C. Lai, A. Klebanoff and E.M. Bollt, Towards complete detection of unstable periodic orbits in chaotic systems, Physics Letters A, 287: 99-104 (2001)

[6] W.H. Press, S.A. Teukolsky, W.T. Vetterling and B.P. Flannery. Numerical recipes in $C$ : the art of scientific computing, Cambridge university press, 1988, ISBN 0-52143108-5

[7] P. Schmelcher and F. K. Diakonos, General approach to the localization of unstable periodic orbits in chaotic dynamical systems, Physical Review E, Vol 57, no. 3, pp. 2739-2746 (1998)

[8] Y. F. Chang and G. Corliss, Ratio-like and recurrence relation tests for convergence of series, J. Inst. Maths. Appl., 25: 349-359 (1980)

[9] http://www.maths.surrey.ac.uk/personal/st/J.Deane/pdf/fastode.pdf

[10] M. Abramowitz and I.A. Stegun, Handbook of mathematical functions, Dover publications Inc., New York, 1972, ISBN 0-486-61272-4

[11] L. Marsh, Nonlinear dynamics of the RL-diode circuit, PhD thesis, Department of Mathematics and Statistics, University of Surrey (submitted 2005)

[12] M.V. Bartuccelli, J.H.B. Deane, G. Gentile and L. Marsh, Invariant sets for the varactor equation, accepted for Proc. Roy. Soc. London, Series A (2005) 
[13] K.B. Blyuss, Chaotic behaviour of solutions to a perturbed Korteweg-de Vries equation, Reports in Mathematical Physics, 49: 29-38 (2002) 